\title{
ANALISIS DE LAS ESTADÍSTICAS DE PRODUCCIÓN DE CARNE Y SEMILLA DE PAICHE Arapaima gigas EN LORETO Y UCAYALI (PERÚ)
}

\author{
Miriam Adriana ALVAN-AGUILAR ${ }^{1}$, Fred William CHU-KOO', Gabriela Cristina BALUARTE MONGE ${ }^{2}$, \\ Luis Ángel COLLADO PANDURO ${ }^{3}$ y Daniel Abelardo VELARDE RÍOS ${ }^{3}$
}

1 Instituto de Investigaciones de la Amazonía Peruana - IIAP. Programa de Investigación para el Uso y Conservación del Agua y sus Recursos - AQUAREC. Centro de Investigaciones Fernando Alcántara Bocanegra - CIFAB. Carretera IquitosNauta Km. 2.5. San Juan Bautista, Maynas, Loreto, Perú. E-mail:malvan@iiap.org.pe

2 Gobierno Regional de Loreto. Dirección Regional de la Producción de Loreto. Dirección de Acuicultura. Av. Ramírez Hurtado 645. Iquitos, Loreto, Perú.

3 Gobierno Regional de Ucayali. Dirección Regional de la Producción de Ucayali. Dirección de Pesquería. Jirón Tacna 826. Pucallpa, Ucayali, Perú.

El paiche, Arapaima gigas, es el segundo pez escamado más grande que habita en aguas dulces después del pez lagarto norteamericano y el sexto entre todos los peces de agua dulce del mundo. Puede alcanzar hasta 3 metros de longitud, pesar 250 kg (Imbiriba, 1994; Salvo \& Val, 1990) y se caracteriza por ser altamente especializado debido a que presenta respiración aérea obligatoria, que lo hace menos dependiente del oxígeno disuelto en el agua; pero que obliga al individuo a alcanzar la superficie en intervalos regulares, para captar el aire atmosférico (Sánchez, 1961; Salvo \& Val, 1990; Castello \& Stewart, 2010).

De acuerdo a Rebaza et al. (1999), esta especie ha demostrado tener un gran potencial para la piscicultura, debido a su rusticidad y fácil adaptación a las condiciones de cultivo, un buen desempeño zootécnico en ambientes controlados, alto valor en el mercado local, así como el poseer una carne de excelente sabor y calidad nutricional. Se reproduce en condiciones de cultivo, alcanza 10 kilos de peso al año; tiene un rendimiento en carne de casi $57 \%$, y condiciones óptimas para la preparación de productos con valor agregado (carne, escamas y cuero).

En términos de mercado externo, países como Estados Unidos, Alemania, Suiza, Chile, España, Holanda y Emiratos Árabes Unidos han mostrado cierto interés en la carne de paiche (Udewald, 2005; Mueller, 2006; Chu-Koo \& Alcántara, 2009; ChuKoo et al., 2012; Schaefer et al., 2012). Productores de Iquitos, Yurimaguas y Pucallpa, vienen realizando envíos de carne a Estados Unidos y España (Jorge Moya Cañas, Gerente de Amazon Fish Products -comunicación personal) y desde el año 2014 a Holanda y Emiratos Árabes Unidos, respectivamente, a raíz de la difusión del producto en ferias alimentarias con apoyo de Promperú (Blgo.
Alberto Vásquez, comunicación personal). Por tanto, el desarrollo del cultivo del paiche (paichicultura), además se convierte en una estrategia para contribuir a la conservación de la especie -actualmente en el Apéndice II del CITESatenuando la presión de pesca en los ambientes naturales de los adultos y alevinos de paiche cada vez más sobreexplotados.

El presente trabajo, tiene como objetivo analizar la evolución reciente de las estadísticas de producción de semilla y carne de paiche en la región Loreto y Ucayali; tomando como base, la información estadística de las Direcciones Regionales de la Producción (DIREPRO) de ambas regiones. Adicionalmente, se presentan datos de comercialización y exportación de productos derivados del paiche del Ministerio de la Producción.

\section{LEVANTE DE SEMILLA DE PAICHE EN LORETO Y UCAYALI}

Según registros de la DIREPRO Loreto, el levante de alevinos de paiche en el periodo 2010 2016 fue de 545,952 ejemplares; mientras que la región Ucayali reportó el levante de 278,798 alevinos en el mismo periodo. En términos globales, ambas regiones levantaron 824,750 alevinos, haciendo una media de levante anual de 117,821 alevinos. En términos porcentuales, Loreto fue el responsable del $66.2 \%$ de la semilla registrada entre el 2010 - 2016, mientras que Ucayali produjo el $33.8 \%$ restante.

Evaluando las estadísticas año por año, observamos que el levante total de semilla en Loreto es fluctuante. El 2010 alcanzó los 94,564 alevinos, bajó a 48,888 ejemplares el 2012; hasta recuperarse y alcanzar 117,465 ejemplares en el 2016. El caso de 
Ucayali, presenta una tendencia creciente. El 2010 se reporta el levante de 1,823 alevinos de paiche, incrementándose a 50,018 en el 2013, presentándose una disminución de alrededor de 22,648 ejemplares el 2014, para luego recuperar el impulso creciente hasta lograr 76,825 alevinos levantados el 2015 (Figura 1).

Es relevante hacer notar que en el 2010 Loreto ya superaba los 90,000 alevinos levantados, cuando en
Ucayali las experiencias en el manejo de la reproducción de paiche en estanques se encontraban en niveles incipientes. Es así que en Ucayali se observa una tendencia creciente en los años de análisis, en función al ajuste de las experiencias logradas. En Loreto en cambio, se destaca el buen manejo de la reproducción casi desde el inicio de la actividad.

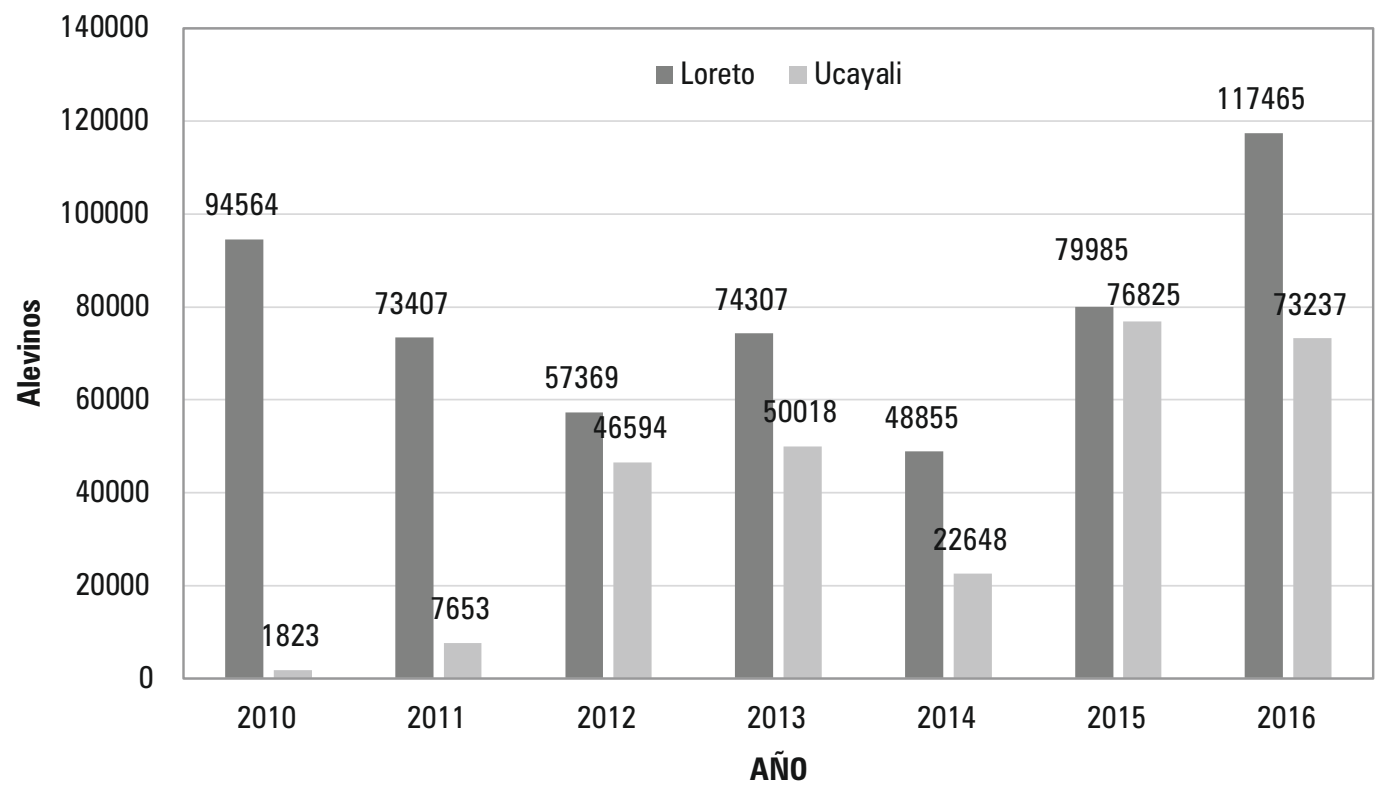

Figura 1. Levante de alevinos de paiche en las regiones amazónicas de Loreto y Ucayali en el periodo 2010 - 2016. Fuente: Elaboración propia con datos de las DIREPRO Loreto y Ucayali.

COMERCIALIZACIÓN DE ALEVINOS DE PAICHE COMO PECES ORNAMENTALES EN LORETO Y UCAYALI

Anualmente, una significativa parte de la producción de semilla de paiche obtenida en Loreto es destinada a la exportación como peces ornamentales. En términos generales, ambas regiones exportaron en el periodo 2007 - 2016 un total de 328,702 alevinos; de los cuales, Loreto exportó 311,917 mientras que Ucayali registró la exportación de 16,785 alevinos el 2015.

En la Figura 2, se presentan las estadísticas de exportación de alevinos como peces ornamentales de la Región Loreto durante el periodo 2007 - 2016. Comparándola con el levante de alevinos registrada en dichos años, los niveles de comercialización de alevinos en Loreto son sobresalientes, pero es notorio que aún queda un número importante de crías para la producción de carne en el territorio nacional.

En la actualidad, alrededor de 12 empresas acuaristas de Iquitos se dedican a servir de intermediarios en este comercio, comprando miles de alevinos a los piscicultores locales para luego enviarlas al exterior. Según registros de la DIREPRO Loreto, el principal destino de los alevinos exportados el 2016 fue la provincia de Hong Kong (República Popular China), con 44,639 ejemplares, es decir el $81.15 \%$ de lo exportado ese año. En la Tabla 1 se detallan las empresas que exportaron alevinos de paiche el año 2016. 


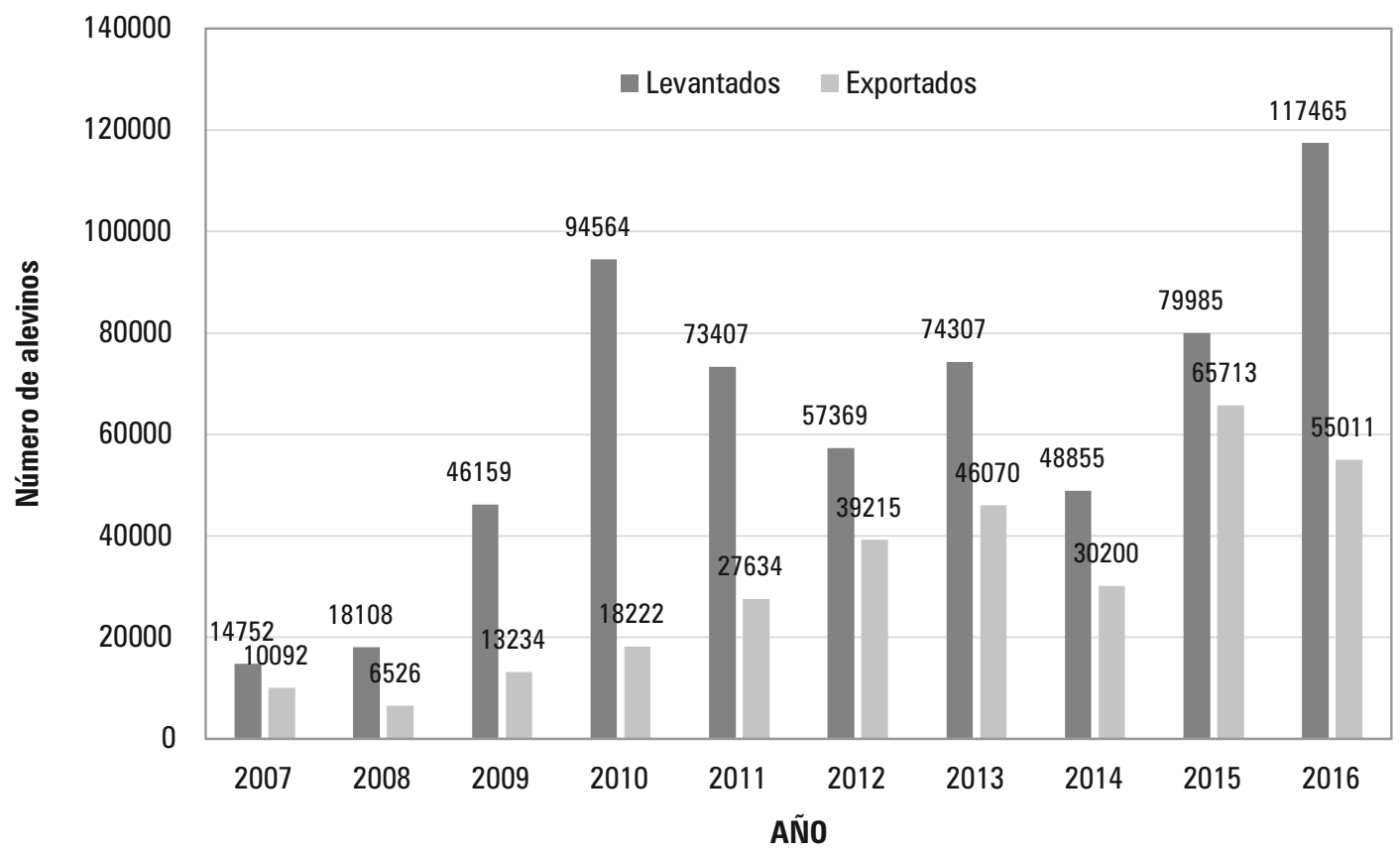

Figura 2. Levante y exportación de alevinos de paiche (Arapaima gigas) desde Loreto en el periodo 2007 - 2016. Fuente: Elaboración propia con datos de la DIREPRO Loreto.

Tabla 1. Empresas acuaristas de lquitos que registraron envíos de alevinos de paiche (Arapaima gigas) a clientes extranjeros. Fuente: DIREPRO Loreto.

\begin{tabular}{llr}
\hline N $^{\mathbf{2}}$ & \multicolumn{1}{c}{ Nombre o Razón Social } & Número Alevinos \\
\hline 1 & Aquarium San Pedro & 1,956 \\
2 & Acuario Valentina EIRL & 7,004 \\
3 & Acuatrade SAC & 6,844 \\
4 & Amazon Tropical Aquarium & 3,375 \\
\hline 5 & Bio Industrial Amazonas SAC & 122 \\
6 & Peruvian Rivers EIRL & 2,013 \\
\hline 7 & Acuario Milagritos EIRL & 16,673 \\
8 & Aquarium Panduro EIRL & 150 \\
\hline 9 & MF Tropical Fish Aquarium EIRL & 12,162 \\
\hline 10 & Riverland Peru & 343 \\
\hline 11 & JR Tropical Fish Amazonas Peru EIRL & 10 \\
\hline 12 & MF Turtles \& Tropical Fish EIRL & 4,359 \\
\hline & TOTAL & 55,011 \\
\hline
\end{tabular}




\section{PRODUCCIÓN DE CARNE DE PAICHE EN LORETO Y UCAYALI}

El gradual incremento de la oferta de semilla y la aparición de una amplia gama de alimentos balanceados comerciales (Purina, Nutrimix, Aquatech y Nicovita) destinados a la alimentación del paiche, han permitido la incursión de inversionistas en la producción de esta especie en Yurimaguas, Iquitos y Pucallpa. Entre los años 2010 al 2016, se obtuvieron 1,046.7 TM de carne de paiche en Loreto, siendo el año 2011 el de mayor producción, con 421.2 TM. En Ucayali, la cosecha de paiche en el periodo 2010 - 2016 fue de 186.3 TM, siendo también el 2011, el año de mayor producción con $74 \mathrm{TM}$ (Figura 3 ).

A la fecha, el principal productor de carne de paiche en el Perú es la empresa Acuícola Los Paiches S.A.C., localizada en Yurimaguas (Alto Amazonas, Loreto) que entre los años 2011 y 2012 cosechó 750 $\mathrm{TM}$, que fueron comercializadas en supermercados y restaurantes de Lima, así como entre clientes de España y Estados Unidos (Chu-Koo et al., 2012). En el 2016, esta empresa bajó su producción, cosechando solo 77.6 TM de carne; pero se desconocen las causas de ello.

El crecimiento en la producción de carne y de semilla en Loreto y Ucayali fue posible por los siguientes aspectos: a) Incremento significativo en el conocimiento científico de la especie, así como los avances en el desarrollo tecnológico de su cultivo, aspecto liderado principalmente por el Instituto de Investigaciones de la Amazonía Peruana (IIAP) y empresarios paichicultores quienes vienen ajustando la tecnología de reproducción y producción. Entre los años 2003 y 2014, el IIAP generó valiosa información sobre la especie; desde su manejo en cautiverio (estanques, jaulas y corrales), rusticidad, protocolos de adaptación a dietas secas, determinación de requerimiento proteico en juveniles, desempeño zootécnico en jaulas flotantes, métodos para determinación de sexo, intentos de reproducción inducida, influencia de parámetros ambientales en la reproducción, caracterización genética de poblaciones naturales, repoblamiento, parásitos, desplazamiento en los ambientes naturales, etc.

b) Incursión de productores e inversionistas privados que han fortalecido la paichicultura en Loreto y Ucayali y otras que han expandido la actividad hacia regiones como Piura, Lima y Tumbes. Importantes Consorcios económicos nacionales como el Grupo Hochschild (propietario de Acuícola Los Paiches S.A.C.), el Grupo Favre (Acuicultura de Huaura S.A.C.) y Camposol S.A. (Grupo Marinazul S.A.), son muestras positivas del creciente interés y del futuro de la paichicultura en el país.

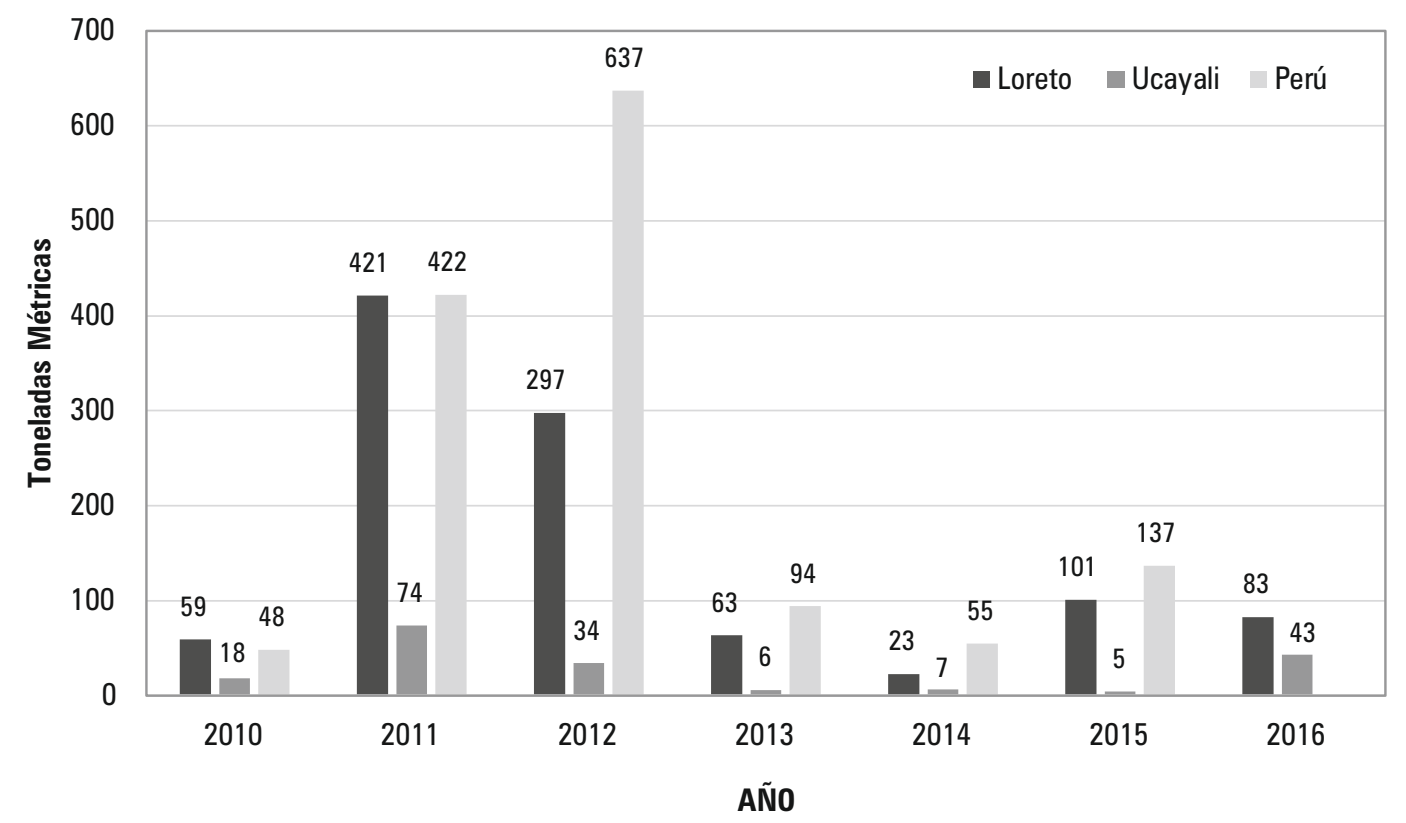

Figura 3. Producción de paiche (Arapaima gigas) en el periodo 2007 - 2016. Fuente: Elaboración propia con datos de PRODUCE y las DIREPRO Loreto y Ucayali. 


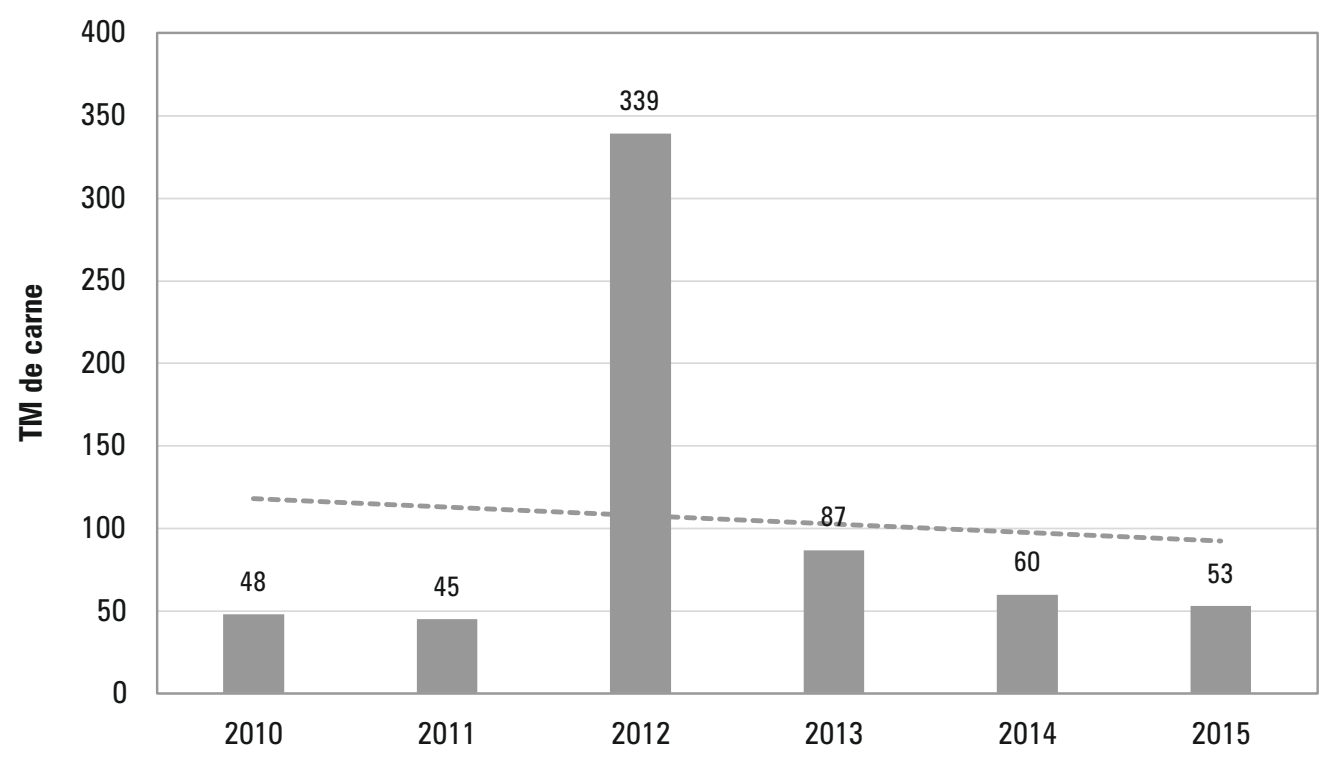

AÑ̃

Figura 4. Venta interna de carne de paiche (TM) entre los años 2010 - 2015. Fuente: Elaboración propia con datos de PRODUCE (2016).

\section{MERCADOS Y COMERCIALIZACIÓN}

Contrario a lo esperado, el mercado nacional mostró ser un espacio interesante para la comercialización de la carne de A. gigas, aunque aún no lo suficientemente grande para darle el impulso a la actividad. Si bien el paiche es considerado un producto eminentemente de exportación, los datos oficiales de PRODUCE (2016), indican que el 47.4 $\%$ de la carne proveniente de acuicultura entre los años 2010 - 2015 (poco más de $632 \mathrm{TM}$ ), fueron comercializados en el mercado interno (Figura 4).

En Ucayali, Collado (2016) reporta que 68.3 TM de paiche proveniente de acuicultura fueron comercializados en los mercados de Pucallpa y Lima entre los años 2015 y agosto de 2016 . Se proyecta una producción de $60 \mathrm{TM}$ de carne de paiche para ser comercializados durante los primeros meses del 2017, por un valor estimado de 2.18 millones de soles.

En cuanto al mercado externo, Mueller (2006), puso en evidencia una demanda potencial por la carne de paiche en Estados Unidos, Alemania y Suiza. En Estados Unidos, un estudio publicado por Schaefer et al. (2012) menciona que el paiche tiene todas las características necesarias para su producción acuícola y que tiene potencial para el mercado norteamericano e internacional, por su valor nutricional y culinario. No es casualidad entonces que el principal destino de la carne de paiche sea justamente los Estados Unidos de Norteamérica, adonde se han exportado 284 TM en el periodo 2010-2015; por un monto total de 4.02 millones de dólares americanos (PRODUCE, 2016).

En total, el Perú exportó en el periodo 2010 2015 , un total de $302 \mathrm{TM}$ de productos derivados del paiche (Figura 5), generándose poco más de 4.44 millones de dólares americanos en divisas (PRODUCE, 2016).

\section{EL FUTURO DE LA PAICHICULTURA EN EL PERÚ}

Según registros actualizados de la DIREPRO Loreto, dicha región cuenta con poco más de 900 hectáreas de infraestructura piscícola formalmente registrada. Si en el mediano plazo se destinase el $25 \%$ de esta capacidad instalada al cultivo intensivo de paiche, Loreto podría producir unas $2250 \mathrm{TM}$ de carne anualmente (10 ton/ha), convirtiéndose en un producto bandera de la acuicultura loretana. Por otro lado, un detallado diagnóstico presentado en el "I Foro para la Promoción y Puesta en Valor del Paiche 


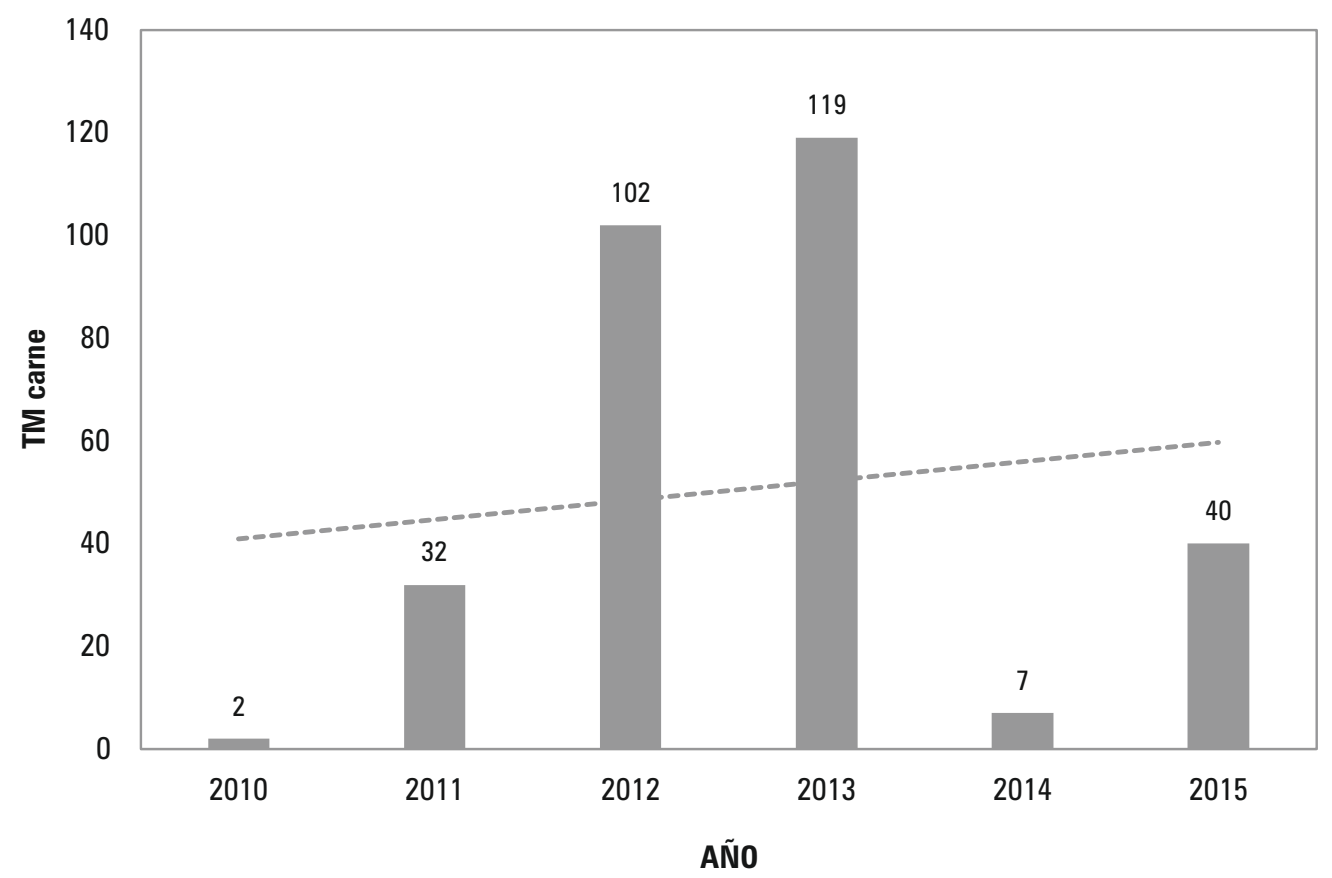

Figura 5. Exportaciones peruanas de carne de paiche (TM) entre los años 2010 - 2015. Fuente: Elaboración propia con datos de PRODUCE (2016).

en la Región Ucayali”, realizado en Pucallpa en setiembre de 2016, indica que existen 13 ha de espejo de agua dedicados a la paichicultura en dicha región (Collado, 2016), lo que indica que potencialmente pueden producir $130 \mathrm{TM} / \mathrm{año}$, que supone que entre ambas regiones amazónicas se alcanzarían los 2380 TM/año.

Para llegar a esos niveles de producción, se necesitará una provisión de 240 a 250 mil alevinos/campaña (en conjunto, Loreto y Ucayali levantaron 190,702 alevinos el 2016). Además, se necesitarán plantas de procesamiento y logística de cadenas de frío certificadas, así como alimento balanceado de garantizada calidad con precios más competitivos que los actuales.

Es obvio que para llegar a ello, se requerirá de una mayor intervención del sector estatal (PRODUCE, ITP, SANIPES, FONDEPES, CITEs, IIAP, MINAM, MINCETUR, GOREs y GOLOs) y privado en incrementar significativamente la inversión en áreas como la investigación, fomento de la actividad con créditos a intereses preferenciales, seguros de riesgo acuícola, capacitación y asistencia técnica a los productores, modernización y mejoramiento de la infraestructura productiva, plantas y/o cadenas de frío, así como en la implementación de políticas que incentiven y faciliten el fortalecimiento y crecimiento de esta actividad productiva y la búsqueda de mercados para su comercialización.

Entidades como el Ministerio de Comercio Exterior y Turismo (MINCETUR) y el Ministerio de la Producción (PRODUCE), han identificado y priorizado al paiche como un producto de exportación de gran potencial para la acuicultura nacional, habiendo realizado consultorías para tratar de resolver los cuellos de botella tecnológicos y de mercado existentes. El desarrollo de las tecnologías de producción y valor agregado es parte importante de la agenda de PRODUCE, sus entes adscritos y el IIAP; mientras que la identificación de nuevos mercados forma parte de la agenda de programas estatales como Sierra y Selva Exportadora y MINCETUR.

Empresarios dedicados a la paichicultura manifiestan que falta fortalecer varios eslabones de la cadena productiva del paiche. Entre los problemas más importantes mencionan la baja calidad y disponibilidad de alimento balanceado, créditos acuícolas a intereses prohibitivos, reducido tamaño 
del mercado, ausencia de plantas de procesamiento y cadenas de frío con certificación y/o habilitaciones sanitarias en Pucallpa, Yurimaguas e Iquitos e inestabilidad en los precios de venta de la carne por la competencia de criadores informales, que influyen en la rentabilidad del producto final.

\section{BIBLIOGRAFÍA CITADA}

Castello, L. \& Stewart, D.J. 2010. Assessing CITES nondetriment finding procedures for Arapaima in Brazil. Journal of Applied Ichthyology, 26: 49-56.

Chu-Koo, F. \& Alcántara, B.F. 2009. Cultivo de paiche doméstico. Perspectivas económicas. Pesca Responsable, 57 (marzo-abril): 32-33.

Chu-Koo, F.; Sánchez, S.N.; Perea, S.C.; Panduro, T.D.; Alvan-Aguilar, M.; Alcántara, F.; Rebaza, C.; Tello, S.; Ferrer, R. \& Núñez, J. 2012. Estado actual del cultivo de paiche o pirarucu en el Perú. Infopesca Internacional, 52:21-25.

Collado, P.L.A. 2016. Diagnóstico de la producción del paiche en la Región Ucayali. Presentación Power Point realizada en el "I Foro para la Promoción y Puesta en Valor del Paiche en la Región Ucayali”. 29 de setiembre de 2016. Pucallpa.

Imbiriba, E. 1994. Reproducão, larva e alevinagem do pirarucu (Arapaima gigas). EMBRAPACPATU. Recomendações básicas, 26: 1-4 p.
Ministerio de la Producción. 2016. Anuario estadístico de Pesca y Acuicultura 2016. LimaPerú. $196 \mathrm{p}$.

Mueller, O. 2006. Arapaima gigas Market Study. Current status of Arapaima global trade and perspectives on the Swiss, French and UK markets. Editado por UNCTAD / BioTrade Facilitation Programme.

Rebaza, A.M.; Alcántara, B.F. \& Valdivieso, M. 1999. Manual de piscicultura del paiche (Arapaima gigas Cuvier). Tratado de Cooperación Amazónica. Secretaria pro tempore. $71 \mathrm{p}$.

Salvo, R.H. \& Val, A.L. 1990. O gigante das àguas doces. Ciência Hoje, 11(64): 10-13.

Sánchez, J. 1961. El paiche, gigante aspectos de su historia natural, ecología y aprovechamiento. Ministerio de Agricultura. 48 p.

Schaefer, F.; Kloas, W. \& Wurtz, S. 2012. Arapaima: Candidate for intensive freshwater culture. Global Aquaculture Advocate (noviembrediciembre):50-51.

Udewald, R. 2005. Potencial de peces amazónicos en el mercado alemán: paiche, gamitana y dorado. Editado por PNPB-PROMPEX, Perú.

Recibido: 21 de Setiembre del 2016

Aceptado para publicación: 17 de Noviembre del 2016 
